J. Korean Math. Soc. 48 (2011), No. 5, pp. 1083-1100

http://dx.doi.org/10.4134/JKMS.2011.48.5.1083

\title{
SPACELIKE MAXIMAL SURFACES, TIMELIKE MINIMAL SURFACES, AND BJÖRLING REPRESENTATION FORMULAE
}

Young Wook Kim, Sung-Eun Koh, Heayong Shin, and Seong-Deog Yang

\begin{abstract}
We show that some class of spacelike maximal surfaces and timelike minimal surfaces match smoothly across the singular curve of the surfaces. Singular Björling representation formulae for generalized spacelike maximal surfaces and for generalized timelike minimal surfaces play important roles in the explanation of this phenomenon.
\end{abstract}

\section{Introduction}

Consider the graph of

$$
t(x, y)=\log \cosh x-\log \cosh y
$$

in the Lorentz-Minkowski three-space $\mathbb{L}^{3}$ equipped with coordinates $(x, y, t)$ and metric $d x^{2}+d y^{2}-d t^{2}$. In [7] it is introduced as the unique, up to homothety and translation, spacelike maximal surface in $\mathbb{L}^{3}$ which is the graph of a function of the form $t=f(x)+g(y)$. Being spacelike, the graph is considered only over the region $\left\{(x, y): \tanh ^{2} x+\tanh ^{2} y<1\right\}$.

While looking at the Figure 1 of the equation (1.1), we simply questioned 'what is the character of the surface if $\tanh ^{2} x+\tanh ^{2} y>1$ ?' It turns out that the surface is timelike there and the mean curvature still vanishes. This is one of the simplest examples where a spacelike maximal surface and a timelike minimal surface match smoothly across their singularities. Another such example is the matching of the spacelike elliptic helicoid and the timelike elliptic helicoid, whose formulae appear in Examples 2.8 and 2.9. See Figure 3.

The main purpose of this article is to discuss what kind of spacelike maximal surfaces and timelike minimal surfaces can match smoothly. This is an

Received June 24, 2010; Revised October 7, 2010.

2010 Mathematics Subject Classification. 53A35, 53B30.

Key words and phrases. spacelike maximal surface, timelike minimal surface, Björling representation formula.

The first named author was supported by NRF 2009-0086794 and a Korea University Grant. The second named author was supported by NRF 2009-0086794 and NRF 20090086441. The last named author was partly supported by a Korea University Grant. 


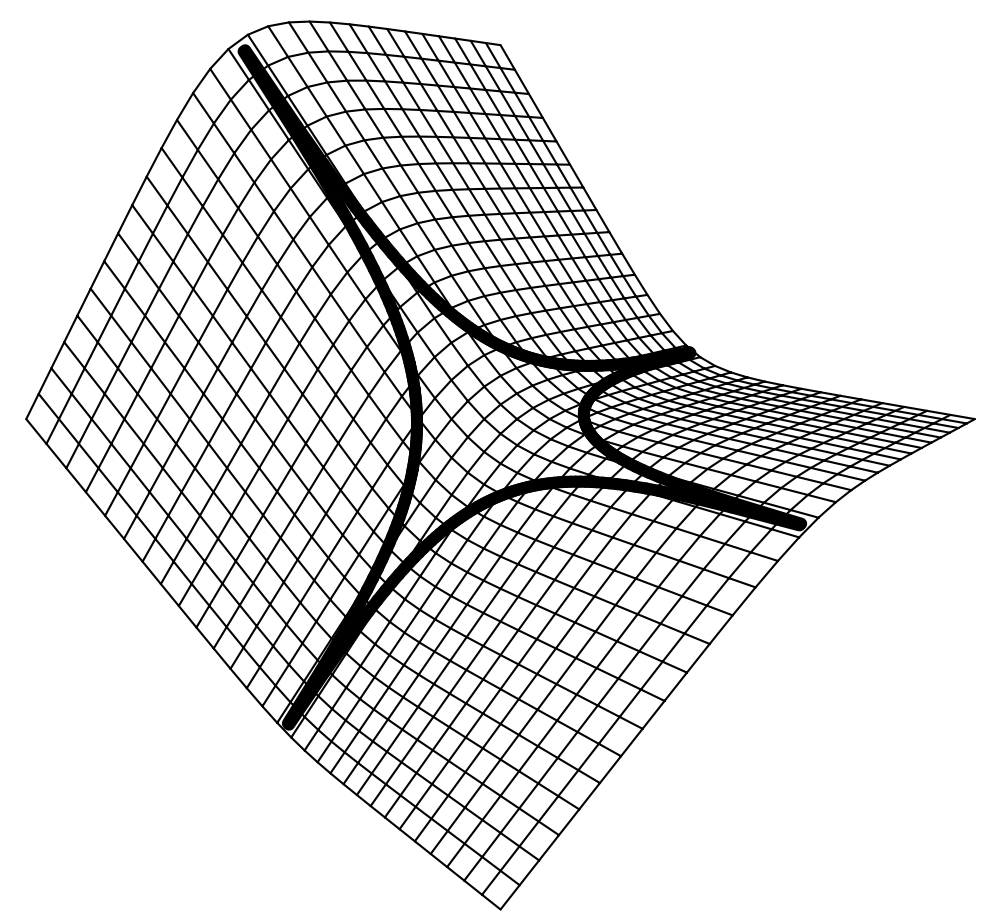

FiguRE 1. Lorentzian Scherk type surface

interesting question because spacelike maximal surfaces and timelike minimal surfaces have been studied in completely different contexts. In particular, neither the Weierstrass type representation formula for spacelike maximal surface by Kobayashi [13] nor the Weierstrass type representation formula for timelike minimal surface by Minor [15] cannot yield the entire Scherk type surface in (1.1).

In achieving our goals, we develop regular and singular Björling representation formulae for generalized timelike minimal surfaces, which play important roles in our analysis of the aforementioned phenomenon. Note that regular and singular Björling formulae for spacelike maximal surfaces have been already studied in several articles $[3,12,16]$.

This article is organized as follows: In Section 2, we recall some known facts and develop basic results relevant to our goal. In Section 3, we develop regular and singular Björling representation formulae for timelike minimal surfaces. In Section 4, we investigate the reflection principles of timelike minimal surfaces. Finally we show in Section 5 that the smooth matching of proper pairs of a generalized spacelike maximal surface and a generalized timelike minimal surface always happen within a certain class of such surfaces. 


\section{Preliminaries and basic results}

\subsection{Generalized spacelike maximal surfaces}

We recall a few known facts: Consider a $C^{2}$ map y $: \mathcal{U} \subset \mathbb{R}^{2} \rightarrow \mathbb{L}^{3}$ with the property that

$$
\left\langle\mathbf{y}_{u}, \mathbf{y}_{u}\right\rangle=\left\langle\mathbf{y}_{v}, \mathbf{y}_{v}\right\rangle \neq 0, \quad\left\langle\mathbf{y}_{u}, \mathbf{y}_{v}\right\rangle=0
$$

Then the mean curvature of $\mathbf{y}$ vanishes if and only if

$$
\mathbf{y}_{u u}+\mathbf{y}_{v v}=0 \text {. }
$$

If we allow singularities, then we obtain the following definition of the generalized spacelike maximal surfaces. For examples of the generalized maximal surfaces, see [9].

Definition 2.1 ([7]). Let $S$ be a Riemann surface and $\mathbf{y}=(x, y, t): S \rightarrow$ $\mathbb{L}^{3}$ be nonconstant and harmonic. Suppose that at any point $p$ of $S$ there exists a (complex) coordinate neighborhood $(U, z)$ such that the complexioned derivatives $\phi_{1}=\partial x / \partial z, \phi_{2}=\partial y / \partial z, \phi_{0}=\partial t / \partial z$ satisfy

$$
\begin{aligned}
\phi_{1}^{2}+\phi_{2}^{2}-\phi_{0}^{2} & =0 \\
\left|\phi_{1}\right|^{2}+\left|\phi_{2}\right|^{2}-\left|\phi_{0}\right|^{2} & \neq 0 \quad \text { (not identically zero). }
\end{aligned}
$$

Then $(\mathbf{y}, S)$ is said to be a generalized spacelike maximal surface.

A generalized spacelike maximal surface $\mathbf{y}: \mathcal{M} \rightarrow \mathbb{L}^{3}$, where $\mathcal{M}$ is a Riemann surface, can be written locally as

$$
\mathbf{y}(z)=\left(x_{0}, y_{0}, t_{0}\right)+\operatorname{Re} \int_{z_{0}}^{z}\left(\frac{1}{2}\left(g^{-1}+g\right), \frac{i}{2}\left(g^{-1}-g\right), 1\right), \quad z \in \mathcal{U}
$$

for some meromorphic function $g$ and a holomorphic one-form $d h$ on some domain $\mathcal{U} \subset \mathbb{C}$ such that the poles of $g$ with order $m$ are zeros of $d h$ of order at least $m$. The metric is $d s^{2}=\frac{1}{4}\left(|g|^{-1}-|g|\right)^{2}|d h|^{2}$, and (2.4) is equivalent to $|g| \not \equiv 1$.

Like minimal surfaces in $\mathbb{E}^{3}$, spacelike maximal surfaces admit regular Björling representation formula, $c f$. [3]. It turns out that generalized spacelike maximal surfaces admit singular Bjölring representation formula.

Theorem 2.2 (Singular Bjölring representation formula for generalized spacelike maximal surfaces [12]). Given an analytic null curve $\gamma:(a, b) \rightarrow \mathbb{L}^{3}$ and an analytic null vector field $\mathcal{L}:(a, b) \rightarrow \mathbb{L}^{3}$ such that $\gamma^{\prime}(u) \perp \mathcal{L}(u)$ and that at least one of $\gamma^{\prime}$ and $\mathcal{L}$ does not vanish identically,

$$
\mathbf{y}(u, v):=\frac{1}{2}(\gamma(u+i v)+\gamma(u-i v))-\frac{i}{2} \int_{u-i v}^{u+i v} \mathcal{L}(t) d t
$$

is a generalized spacelike maximal surface which contains $\gamma$ as a singular curve and $\mathcal{L}(u)$ as a coordinate vector field along $\gamma(u)$. 


\subsection{Generalized timelike minimal surfaces in $\mathbb{L}^{3}$}

Consider a $C^{2}$ map $\mathbf{x}: \mathcal{U} \subset \mathbb{R}^{2} \rightarrow \mathbb{L}^{3}$ with the property that

$$
\left\langle\mathbf{x}_{u}, \mathbf{x}_{u}\right\rangle=-\left\langle\mathbf{x}_{v}, \mathbf{x}_{v}\right\rangle \neq 0, \quad\left\langle\mathbf{x}_{u}, \mathbf{x}_{v}\right\rangle=0 .
$$

The mean curvature of $\mathbf{x}$ vanishes if and only if

$$
\mathbf{x}_{u u}-\mathbf{x}_{v v}=0 .
$$

If we set new coordinates $\alpha=u+v, \beta=u-v$, then $\left\langle\mathbf{x}_{\alpha}, \mathbf{x}_{\alpha}\right\rangle=-\left\langle\mathbf{x}_{\beta}, \mathbf{x}_{\beta}\right\rangle=$ $0,\left\langle\mathbf{x}_{\alpha}, \mathbf{x}_{\beta}\right\rangle \neq 0$, and the mean curvature $H$ vanishes if and only if $\mathbf{x}_{u v}=0$. From this, the first half of the following theorem follows immediately.

Theorem $2.3([15])$. If $\mathcal{X}(t)$ and $\mathcal{Y}(t)$ are null curves in $\mathbb{L}^{3}$ such that $\mathcal{X}^{\prime}(x)$ and $\mathcal{Y}^{\prime}(y)$ are perpendicular to each other for all $x$ and $y$, then

$$
\mathcal{X}(x)+\mathcal{Y}(y)
$$

is a timelike minimal surface. Conversely, any timelike minimal surface may be written locally as (2.8) for some two null curves.

Like spacelike maximal surfaces, there are a large number of timelike minimal surfaces with singularities, so we enlarge the class of timelike minimal surfaces:

Definition 2.4. Let $S$ be a $C^{2}$ 2-manifold and $\mathrm{x}: S \rightarrow \mathbb{L}^{3}$ be a nonconstant map. Suppose that at any point of $S$ there exists a coordinates system $(\mathcal{U},(u, v))$ such that
(1) $\left\langle\mathbf{x}_{u}, \mathbf{x}_{u}\right\rangle \equiv-\left\langle\mathbf{x}_{v}, \mathbf{x}_{v}\right\rangle \geq 0,\left\langle\mathbf{x}_{u}, \mathbf{x}_{v}\right\rangle \equiv 0$,
(2) $\mathbf{x}_{u u}-\mathbf{x}_{v v} \equiv 0$,
(3) $\left\langle\mathbf{x}_{u}, \mathbf{x}_{u}\right\rangle=-\left\langle\mathbf{x}_{v}, \mathbf{x}_{v}\right\rangle>0$ almost everywhere on $S$.

Then we call $(\mathbf{x}, S)$ a generalized timelike minimal surface.

An easiest way to construct generalized timelike minimal surfaces is to allow $\mathcal{X}$ and $\mathcal{Y}$ to be linearly dependent in Theorem 2.3 , as we will see later.

We omit $S$ in general in the definition of the generalized timelike minimal surface. Now we distinguish the singularities of $\mathbf{x}$. Fix a coordinates system $(\mathcal{U},(u, v))$ as above and let

$$
\mathcal{A}:=\left\{p \in \mathcal{U}: \mathbf{x}_{u} \text { or } \mathbf{x}_{v} \text { is lightlike }\right\}, \quad \mathcal{B}:=\{p \in \mathcal{U}: d \mathbf{x}(p) \text { vanishes }\} .
$$

Then $V=\mathcal{A} \cup \mathcal{B}$, and $p \in \mathcal{S}$ is a singular point if and only if $p \in \mathcal{A} \cup \mathcal{B}$. We call a point in $\mathcal{B}$ a branch point. In general, $\mathcal{A} \cap \mathcal{B} \neq \emptyset$.

Following [12], we divide $\mathcal{A}$ into three disjoint sets as in the following definition, where $D_{p}(\epsilon)$ is an open disk of radius $\epsilon$ centered at $p$.

Definition 2.5. We call $p \in \mathcal{A}$ a shrinking singular point if there is some $D_{p}(\epsilon) \subset \mathcal{U}$ and a regular embedded curve $\gamma: I \rightarrow D_{p}(\epsilon) \subset \mathcal{U}$ such that $\gamma[I]=D_{p}(\epsilon) \cap \mathcal{A}$ and $X \circ \gamma$ is a single point. If $p$ is a shrinking singular point, we call $\mathbf{x}(p)$ a shrunken singular point, and the germ of $\left(\mathbf{x}, D_{p}(\epsilon)\right)$ a shrunken singularity. 


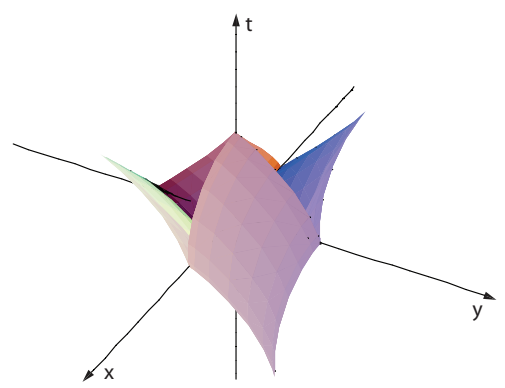

Figure 2. A generalized timelike minimal torus in Example 2.7

We call $p \in \mathcal{A}$ a curvilinear singular point if there is some $D_{p}(\epsilon) \subset \mathcal{U}$ and a regular embedded curve $\gamma: I \rightarrow D_{p}(\epsilon) \subset \mathcal{U}$ such that $\gamma[I]=D_{p}(\epsilon) \cap \mathcal{A}$ and $\mathbf{x} \circ \gamma$ is one-to-one on $I$. If $p$ is a curvilinear singular point, we call the germ of $\left(\mathbf{x}, D_{p}(\epsilon)\right)$ a curvilinear singularity.

We call $p \in \mathcal{A}$ which is neither shrinking nor curvilinear an exotic singular point.

We do not require that the rank of $d \mathbf{x}$ is never zero.

We need to distinguish a special kind of singularities from other curvilinear singularities, whose name is derived from the phenomenon presented in Lemma 4.3.

Definition 2.6. We call a curvilinear singular point $p \in \mathcal{U}$ a folding singular point if it has a neighborhood that can be reparameterized as $\mathbf{x}: D_{0}(\epsilon) \subset$ $\mathbb{R}^{2} \rightarrow \mathbb{L}^{3}$ with $p=0$ such that the singular points are $\{(u, 0):-\epsilon<u<\epsilon\}$ and that $\mathbf{x}_{v}(u, 0) \equiv \overrightarrow{0} . \mathbf{x}\left(D_{0}(\epsilon)\right)$ is called a folded singularity.

Note that there does not exist closed minimal surfaces in $\mathbb{E}^{3}$ nor closed spacelike maximal surfaces in $\mathbb{L}^{3}$ due to the maximum principle. Using Theorem 2.3 , it is easy to construct a generalized timelike minimal torus in $\mathbb{L}^{3}$ (with singularities).

Example 2.7. The curve

$$
\gamma(t):=\left(\frac{1}{2} \sin t+\frac{1}{6} \sin 3 t, \frac{1}{2} \cos t-\frac{1}{6} \cos 3 t, \frac{1}{2} \sin 2 t\right)
$$

is a periodic analytic null curve with four cusps. $\mathbf{x}(u, v):=\gamma(u)+\gamma(v)$ is a generalized timelike minimal surface defined on a torus $\mathbb{R} / 2 \pi \times \mathbb{R} / 2 \pi$.

It would be interesting to see if there exist higher genus examples. 


\subsection{A relation between the spacelike maximal and the timelike min- imal surfaces}

Note that the equations (2.1) and (2.2) for spacelike maximal surfaces are transformed to the equations (2.6) and (2.7) for timelike minimal surfaces via

$$
(u, v) \rightarrow(u,-i v) \text {. }
$$

This is purely formal, but we will see that we can translate some of the well known theories for spacelike maximal surfaces into those for timelike minimal surfaces via the transformation. The transformation (2.9) is called a Wick rotation in physics [14].

\subsection{Basic examples: catenoids and helicoids}

We provide here some explicit description of the catenoids and helicoid in $\mathbb{L}^{3}$. They already appear in [3], [14], but we present them again emphasizing our point of view. To name these surfaces efficiently we use the initials from \{Spacelike, Timelike\}, \{Elliptic, Parabolic, Hyperbolic $\},\{$ Catenoid, Helicoid $\}$. For example, spacelike elliptic catenoid will be simply called SEC, etc.

Example 2.8 (Spacelike catenoids and helicoids [3], [12]).

$$
\begin{aligned}
& \mathbf{y}_{\mathrm{SEC}}(u, v)=(\cos u \sinh v, \sin u \sinh v, v), \\
& \mathbf{y}_{\mathrm{SPC}}(u, v)=\left(v+\frac{v^{3}}{3}-u^{2} v, 2 u v, v-\frac{v^{3}}{3}+u^{2} v\right), \\
& \mathbf{y}_{\mathrm{SHC}}(u, v)=(v, \sin v \sinh u, \sin v \cosh u),
\end{aligned}
$$

are the spacelike elliptic, parabolic and hyperbolic catenoids and

$$
\begin{aligned}
& \mathbf{y}_{\mathrm{SEH}}(u, v)=(\cos u \cosh v, \sin u \cosh v, u), \\
& \mathbf{y}_{\mathrm{SPH}}(u, v)=\left(-u+\frac{u^{3}}{3}-u v^{2},-u^{2}+v^{2},-u-\frac{u^{3}}{3}+u v^{2}\right), \\
& \mathbf{y}_{\mathrm{SHH}}(u, v)=(-u,-\cosh u \cos v,-\sinh u \cos v),
\end{aligned}
$$

are the spacelike elliptic, parabolic and hyperbolic helicoid, respectively.

Example 2.9 (Timelike catenoids and helicoids [14]).

$$
\begin{aligned}
& \mathbf{x}_{\mathrm{TEC}}(u, v)=(\cos u \sin v, \sin u \sin v, v), \\
& \mathbf{x}_{\mathrm{TPC}}(u, v)=\left(v-u^{2} v-\frac{v^{3}}{3}, 2 u v, v+u^{2} v+\frac{v^{3}}{3}\right), \\
& \mathbf{x}_{\mathrm{THC}}(u, v)=(v, \sinh u \sinh v, \cosh u \sinh v),
\end{aligned}
$$

are the timelike elliptic, parabolic and hyperbolic catenoids and

$$
\begin{aligned}
& \mathbf{x}_{\mathrm{TEH}}(u, v)=(\cos u \cos v, \sin u \cos v, u), \\
& \mathbf{x}_{\mathrm{TPH}}(u, v)=\left(-u+\frac{u^{3}}{3}+u v^{2},-u^{2}-v^{2},-u-\frac{u^{3}}{3}-u v^{2}\right),
\end{aligned}
$$




$$
\mathbf{x}_{\mathrm{THн}}(u, v)=(-u,-\cosh u \cosh v,-\sinh u \cosh v) .
$$

are the timelike elliptic, parabolic and hyperbolic helicoid, respectively,
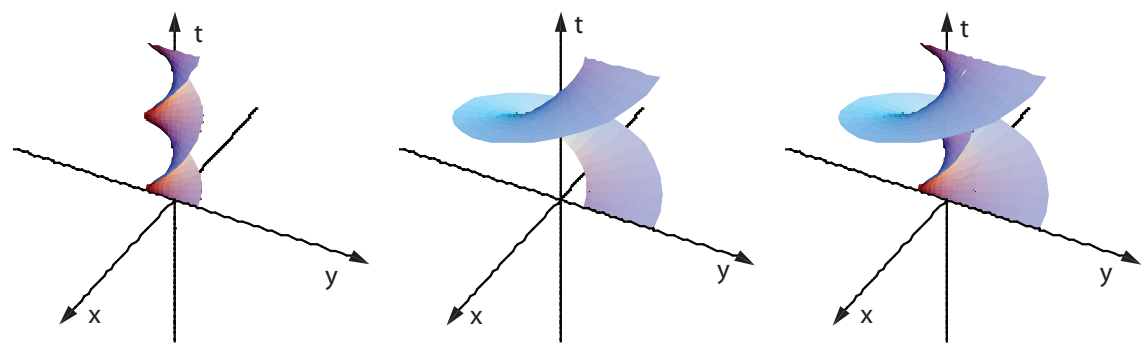

FigurE 3. Timelike elliptic helicoid, spacelike elliptic helicoid, and both of them
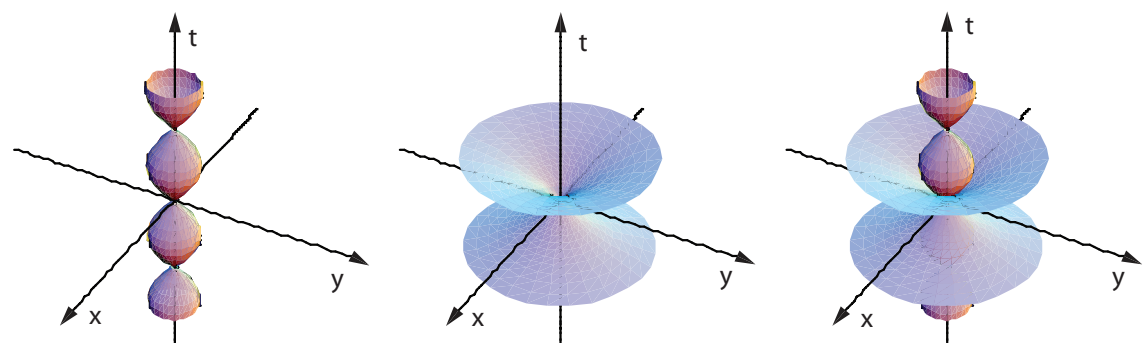

FIguRE 4. Timelike elliptic catenoid, spacelike elliptic catenoid, and both of them

The following is our first encounter of how the Wick rotation (2.9) may be used to relate the spacelike maximal surfaces and the timelike minimal surfaces.

Observation 2.10. For $* \in\{E, P, H\}$, we formally have

$$
\mathbf{x}_{\mathrm{T} * \mathrm{H}}(u, v)=\mathbf{y}_{\mathrm{S} * \mathrm{H}}(u, i v), \quad \mathbf{x}_{\mathrm{T} * \mathrm{C}}(u, v)=-i \mathbf{y}_{\mathrm{S} * \mathrm{C}}(u, i v) .
$$

We will see another instance of the Wick rotation in the next section.

Now we see that the spacelike and the timelike helicoids also match smoothly as was mentioned in Section 1. In fact, they are graphs of the same function like the Lorentzian Scherk type surface as we see in the following lemma.

Lemma 2.11. For timelike and spacelike helicoids,

(1) both $\mathbf{x}_{\mathrm{TEH}}$ and $\mathbf{y}_{\mathrm{SEH}}$ satisfy $y=x \tan t$,

(2) both $\mathbf{x}_{\mathrm{TPH}}$ and $\mathbf{y}_{\mathrm{SPH}}$ satisfy $y=\frac{x-t}{x+t}-\frac{1}{6}(x+t)^{2}$,

(3) both $\mathbf{x}_{\mathrm{THH}}$ and $\mathbf{y}_{\mathrm{SHH}}$ satisfy $y=-t \operatorname{coth} x$.

Here, $x, y, t$ are the components of $\mathbf{x}_{\mathrm{T} * \mathrm{H}}$ and $\mathbf{y}_{\mathrm{S} * \mathrm{H}}$. 
Proof. Direct computations.

The matching property does not hold between spacelike and timelike catenoids, as can be seen immediately from Figure 4.

We now summarize in the following lemma a few of the relations between timelike and spacelike catenoids, and between timelike and spacelike helicoids which are relevant to our study. We omit the proofs since they can be proved by direct calculations with the formulae in Examples 2.8 and 2.9.

Lemma 2.12. Let $* \in\{\mathrm{E}, \mathrm{P}, \mathrm{H}\}$. Then, for spacelike and timelike catenoids

(1) both $\mathbf{x}_{\mathrm{T} * \mathrm{C}}$ and $\mathbf{y}_{\mathrm{S} * \mathrm{C}}$ are rotational surfaces,

(2) both $\mathbf{x}_{\mathrm{T} * \mathrm{C}}$ and $\mathbf{y}_{\mathrm{S} *_{\mathrm{C}}}$ have shrinking singular points on the u-axis,

(3) $\mathbf{x}_{\mathrm{T} * \mathrm{C}}(u,-v)=-\mathbf{x}_{\mathrm{T} * \mathrm{C}}(u, v)$ and $\mathbf{y}_{\mathrm{S} * \mathrm{C}}(u,-v)=-\mathbf{y}_{\mathrm{S} * \mathrm{C}}(u, v)$,

and for spacelike and timelike helicoids

(4) both $\mathbf{x}_{\mathrm{T} * \mathrm{H}}$ and $\mathbf{y}_{\mathrm{S} * \mathrm{H}}$ are ruled surfaces,

(5) both $\mathbf{x}_{\mathrm{T} * \mathrm{H}}$ and $\mathbf{y}_{\mathrm{S} * \mathrm{H}}$ have folding singular points on the u-axis,

(6) $\mathbf{x}_{\mathrm{T} * \mathrm{H}}(u,-v)=\mathbf{x}_{\mathrm{T} * \mathrm{H}}(u, v)$ and $\mathbf{y}_{\mathrm{S} * \mathrm{H}}(u,-v)=\mathbf{y}_{\mathrm{S} * \mathrm{H}}(u, v)$.

(3) and (6) are reflection properties which turn out to be true for any generalized timelike minimal surfaces with a certain kind of singularities, and will be investigated in Section 4.

\section{Björling representation formulae for timelike minimal surfaces}

Given $a, b \in \mathbb{R}$ with $a<b$, we let

$$
\begin{aligned}
\diamond_{a, b} & :=\left\{(u, v) \in \mathbb{R}^{2}: a<u+v<b \text { and } a<u-v<b\right\} \\
I_{a, b} & :=\left\{(u, 0) \in \diamond_{a, b}: a<u<b\right\}
\end{aligned}
$$

in the rest of this article.

Proposition 3.1. Suppose a $C^{2}$ curve $\gamma:(a, b) \rightarrow \mathbb{L}^{3}$, and a $C^{1}$ vector field $\mathcal{L}:(a, b) \rightarrow \mathbb{L}^{3}$ satisfy

(1) $\left\langle\gamma^{\prime}(t), \gamma^{\prime}(t)\right\rangle \equiv-\langle\mathcal{L}(t), \mathcal{L}(t)\rangle$

(2) $\left\langle\gamma^{\prime}(t), \mathcal{L}(t)\right\rangle \equiv 0$

(3) $\gamma^{\prime} \not \equiv \overrightarrow{0}$ or $\mathcal{L} \not \equiv \overrightarrow{0}$, and $\gamma^{\prime} \not \equiv \pm \mathcal{L}$.

Then, the map

$$
\mathbf{x}(u, v):=\frac{1}{2}(\gamma(u+v)+\gamma(u-v))+\frac{1}{2} \int_{u-v}^{u+v} \mathcal{L}(s) d s,
$$

on $\nabla_{a, b}$ is a generalized timelike minimal surface. Furthermore,

$$
\mathbf{x}(u, 0)=\gamma(u), \quad \mathbf{x}_{v}(u, 0)=\mathcal{L}(u) \quad \forall(u, 0) \in I_{a, b} .
$$

That is, $\mathbf{x}$ contains $\gamma$ and has $\mathcal{L}$ as the coordinate vector field. 
Proof. It is straightforward to see that

$$
\begin{aligned}
& 2 \mathbf{x}_{u}=\left[\gamma^{\prime}(u+v)+\mathcal{L}(u+v)\right]+\left[\gamma^{\prime}(u-v)-\mathcal{L}(u-v)\right], \\
& 2 \mathbf{x}_{v}=\left[\gamma^{\prime}(u+v)+\mathcal{L}(u+v)\right]-\left[\gamma^{\prime}(u-v)-\mathcal{L}(u-v)\right]
\end{aligned}
$$

and that

$$
\left\langle\mathbf{x}_{u}, \mathbf{x}_{u}\right\rangle+\left\langle\mathbf{x}_{v}, \mathbf{x}_{v}\right\rangle=0, \quad\left\langle\mathbf{x}_{u}, \mathbf{x}_{v}\right\rangle=0, \quad \mathbf{x}_{u u}-\mathbf{x}_{v v}=0 .
$$

So $\mathbf{x}$ is a generalized timelike minimal surface. The rest of the conclusions are trivial.

Remark 3.2 (d'Alembert's solution of the one dimensional wave equation). Given $f, g:(a, b) \rightarrow \mathbb{R}$

$$
x(u, v):=\frac{1}{2}(f(u+v)+f(u-v))+\frac{1}{2} \int_{u-v}^{u+v} g(s) d s
$$

is the unique solution on $\nabla_{a, b}$ of the Cauchy problem $x_{u u}-x_{v v}=0$ on $\nabla_{a, b}$, $x(u, 0)=f(u), x_{u}(u, 0)=g(u)$ for all $(u, 0) \in I_{a, b}$ (See [4] for example).

Theorem 3.3 (Regular Björling representation formula). Let $\gamma$ be a spacelike or timelike $C^{2}$ curve with nonvanishing velocity, and let $N$ be a spacelike $C^{1}$ vector field of unit length such that $\left\langle\gamma^{\prime}, N\right\rangle \equiv 0$. Then, there exists a timelike minimal surface which contains $\gamma$ and whose unit normal along $\gamma$ is $N$.

Proof. Take $\mathcal{L}=N \times \gamma^{\prime}$ and apply Proposition 3.1.

If $\gamma$ is a null curve and $N$ is a spacelike vector field of unit length, then $N \times \gamma^{\prime}$ is equal to $\gamma^{\prime}$ or $-\gamma^{\prime}$. The $\mathbf{x}(u, v)$ in (3.1) with this $\gamma$ and $\mathcal{L}:=N \times \gamma^{\prime}$ is in fact $\gamma(u+v)$ or $\gamma(u-v)$. For a regular Björling representation formula with a null curve as the prescribed curve, we use T. K. Milnor's representation formula directly:

Lemma 3.4. Suppose $\gamma:(a, b) \rightarrow \mathbb{L}^{3}$ is a $C^{2}$ null curve. Then, there exists a timelike minimal surface $\mathbf{x}:(a, b) \times(-\epsilon, \epsilon) \rightarrow \mathbb{L}^{3}$ such that $\mathbf{x}(u, 0)=\gamma(u)$ and that $\mathbf{x}_{u}(u, 0)$ and $\mathbf{x}_{v}(u, 0)$ are linearly independent for any $u \in(a, b)$.

Proof. Let $\delta:(-\epsilon, \epsilon) \rightarrow \mathbb{L}^{3}$ be an arbitrary $C^{2}$ null curve with $\delta(0)=\overrightarrow{0}$ such that $\delta^{\prime}(v)$ and $\gamma^{\prime}(u)$ are linearly independent for all $u, v$. Then $\mathbf{x}(u, v):=$ $\gamma(u)+\delta(v)$ satisfies the required properties by Theorem 2.3.

Note that $\mathbf{x}_{v}(u, 0)$ is constant if $\mathbf{x}(u, v):=\gamma(u)+\delta(v)$.

The situation in the Proposition 3.1 applies even if both $\gamma$ and $\mathcal{L}$ are null and we obtain the following theorem, which is of greater interest to us than the above regular Björling representation formula since it provides a key to understanding the matching property of the spacelike maximal surfaces and the timelike minimal surfaces.

Theorem 3.5 (Singular Björling representation formula). Suppose a $C^{2}$ curve $\gamma:(a, b) \rightarrow \mathbb{L}^{3}$, and a $C^{1}$ vector field $\mathcal{L}:(a, b) \rightarrow \mathbb{L}^{3}$ satisfy 
(1) $\left\langle\gamma^{\prime}(t), \gamma^{\prime}(t)\right\rangle \equiv-\langle\mathcal{L}(t), \mathcal{L}(t)\rangle=0$,

(2) $\left\langle\gamma^{\prime}(t), \mathcal{L}(t)\right\rangle \equiv 0$

(3) $\gamma^{\prime} \not \equiv \overrightarrow{0}$ or $\mathcal{L} \not \equiv \overrightarrow{0}$, and $\gamma^{\prime} \not \equiv \mathcal{L}$.

Then there exists a unique generalized timelike minimal surface $\mathbf{x}: \diamond_{a, b} \rightarrow \mathbb{L}^{3}$ which satisfies

$$
\mathbf{x}(u, 0)=\gamma(u), \quad \mathbf{x}_{v}(u, 0)=\mathcal{L}(u) \quad \forall(u, 0) \in I_{a, b},
$$

and it actually is

$$
\mathbf{x}(u, v):=\frac{1}{2}(\gamma(u+v)+\gamma(u-v))+\frac{1}{2} \int_{u-v}^{u+v} \mathcal{L}(s) d s .
$$

Proof. The fact that the $\mathbf{x}$ given by (3.2) satisfies all the required properties follows easily from the Proposition 3.1. The uniqueness follows from the uniqueness of the solution of the wave equation on $\diamond_{a, b}$ (cf. Remark 3.2).

Let $\varphi: \tilde{u} \in(c, d) \rightarrow u \in(a, b)$ be a diffeomorphism and let

$$
\tilde{\gamma}(\tilde{u}):=\gamma(\varphi(\tilde{u}))=\gamma(u), \quad \tilde{\mathcal{L}}(\tilde{u}):=\mathcal{L}(\varphi(\tilde{u})) \varphi^{\prime}(\tilde{u}) .
$$

Then the resulting $\tilde{\mathbf{x}}$ and $\mathbf{x}$ by $(3.2)$ with $\tilde{\gamma}(\tilde{u}), \tilde{\mathcal{L}}(\tilde{u})$ and $\gamma(u), \mathcal{L}(u)$, respectively, satisfy

$$
\tilde{\mathbf{x}}(\tilde{u}, \tilde{v})=\mathbf{x}\left(\frac{1}{2}(\varphi(\tilde{u}+\tilde{v})+\varphi(\tilde{u}-\tilde{v})), \frac{1}{2}(\varphi(\tilde{u}+\tilde{v})-\varphi(\tilde{u}-\tilde{v}))\right)
$$

whenever both sides make sense. That is, the resulting timelike minimal surface does not depend upon a parametrization of the interval.

Remark 3.6. It is interesting to compare the singular Björling representation formula (3.2) for generalized timelike minimal surfaces and the singular Björling representation formula (2.5) for generalized spacelike maximal surfaces.

(1) If $\gamma^{\prime}(t) \equiv p m \mathcal{L}$, then map $\mathbf{x}$ given by (3.2) degenerates to $\pm \gamma$. This does not happen with $(2.5)$.

(2) We see that (2.5) is in fact (3.2) with $v$ replaced by $i v$ and $\mathcal{L}$ replace by $i \mathcal{L}$. This is another instance of the Wick rotation (2.9).

(3) In (2.5), both $\gamma$ and $\mathcal{L}$ are required to be analytic. On the other hand, in (3.2), $\gamma$ needs to be only $C^{2}$ and $\mathcal{L}$ needs to be only $C^{1}$. Since there are many pairs of $C^{2} \gamma$ and $C^{1} \mathcal{L}$ such that $\gamma^{\prime}$ and $L$ coincide on a subinterval $J \subset I$ but not on $I \backslash J$, the Björling formulae for timelike surfaces are much more restricted than the Björling formulae for space maximal surfaces.

Note that the two conditions (1), (2) of Theorem 3.5 imply that $\gamma^{\prime}$ is parallel to $\mathcal{L}$. There are two cases which are of some special interests.

Corollary 3.7 (Singular Björling representation formula for folded singularities). If $\mathcal{L} \equiv \overrightarrow{0}$ in addition to the hypotheses of Theorem 3.5, the image of any $(u, 0) \in I_{a, b}$ is a folded singularity of the resulting $\mathbf{x}$. Note that $\mathbf{x}(u, 0)=\gamma(u)$ for any $(u, 0) \in I_{a, b}$. 
Corollary 3.8 (Singular Björling representation formula for shrunken singularities). If $\gamma \equiv \overrightarrow{0}$ in addition to the hypotheses of Theorem 3.5, then the image of any $(u, 0) \in I_{a, b}$ is a shrunken singularity of the resulting $\mathbf{x}$. Note that the image of the entire interval $I_{a, b}$ is a single point.

Remark 3.9. Given $\gamma$ and $\mathcal{L}$ which satisfy the two conditions (1), (2) of Proposition 3.1, define

$$
\mathcal{X}(t):=\frac{1}{2}(\gamma(t)+L(t)), \quad \mathcal{Y}(t):=\frac{1}{2}(\gamma(t)-L(t)),
$$

where $L(t):=\int_{t_{0}}^{t} \mathcal{L}(\tilde{t}) d \tilde{t}$ for some fixed $t_{0}$. Then $\mathcal{X}$ and $\mathcal{Y}$ are null curves and $\mathbf{x}(u, v)$ in $(3.1)$ is equal to $\mathcal{X}(u+v)+\mathcal{Y}(u-v)$. So, if $\left\langle\gamma^{\prime}(t), \gamma^{\prime}(t)\right\rangle=$ $-\langle\mathcal{L}(t), \mathcal{L}(t)\rangle \neq 0$ for all $t$, we recover T. K. Milnor's representation formula.

Example 3.10 (Rotationally invariant generalized timelike minimal surfaces around a timelike, lightlike, spacelike axis, respectively). Let

$$
\begin{aligned}
& \mathcal{L}_{\mathrm{TEC}}(u)=(\cos u, \sin u, 1), \\
& \mathcal{L}_{\mathrm{TPC}}(u)=\left(1-u^{2}, 2 u, 1+u^{2}\right), \\
& \mathcal{L}_{\mathrm{THC}}(u)=(1, \sinh u, \cosh u) .
\end{aligned}
$$

They are rotationally invariant vector fields around a timelike, lightlike, spacelike axis, respectively. Then $(3.2)$ with $\gamma \equiv \overrightarrow{0}$ and $\mathcal{L}=\mathcal{L}_{\mathrm{TEC}}, \mathcal{L}_{\mathrm{TPC}}$, or $\mathcal{L}_{\mathrm{THC}}$ yields

$$
\mathbf{x}_{\mathrm{TEC}}(u, v), \quad \mathbf{x}_{\mathrm{TPC}}(u, v), \quad \mathbf{x}_{\mathrm{THC}}(u, v),
$$

respectively, as in Example 2.9, which are surfaces of revolution.

Note that in the above three examples all the points of the $u$-axis are shrinking singular points.

Example 3.11 (Generalized timelike minimal surfaces invariant with respect to the screw motions around a timelike, lightlike, spacelike axis, respectively). Let

$$
\begin{aligned}
\gamma_{\mathrm{TEC}}(u) & =(\cos u, \sin u, u), \\
\gamma_{\mathrm{TPC}}(u) & =-\left(u-\frac{u^{3}}{3}, u^{2}, u+\frac{u^{3}}{3}\right), \\
\gamma_{\mathrm{THC}}(u) & =-(u, \cosh u, \sinh u) .
\end{aligned}
$$

Then (3.2) with $\gamma=\gamma_{\mathrm{TEC}}, \gamma_{\mathrm{TPC}}, \gamma_{\mathrm{THC}}$ and $\mathcal{L} \equiv \overrightarrow{0}$ yields

$$
\mathbf{x}_{\mathrm{TEH}}(u, v), \quad \mathbf{x}_{\mathrm{TPH}}(u, v), \quad \mathbf{x}_{\mathrm{THн}}(u, v) .
$$

in Example 2.9.

Note that for $\mathbf{x}_{\mathrm{TEH}}, \mathbf{x}_{\mathrm{TPH}}$ and $\mathbf{x}_{\mathrm{THH}}$ all the points of the $u$-axis are folding singular points and that $\partial_{u} \gamma_{\mathrm{T} * \mathrm{C}}(u)$ is the same as $\mathcal{L}_{\mathrm{T} * \mathrm{C}}(u)$ modulo the symmetries of $\mathbb{L}^{3}$.

An immediate consequence of Theorem 3.5 is the following: 
Lemma 3.12. There is no generalized timelike minimal surface which contains (a piece of) a lightlike line as a singular curve.

Proof. Suppose a generalized timelike minimal surface contains a lightlike line. Then we may assume that the surface may be reconstructed from the singular Björling representation formula $(3.2)$ with $\gamma(u)=(u, 0, u)$ and $\mathcal{L}(u)=$ $(\phi(u), 0, \phi(u))$ for some function $\phi$. But the resulting $\mathbf{x}$ is

$$
\mathbf{x}(u, v)=\left(u+\frac{1}{2} \int_{u-v}^{u+v} \phi(s) d s, 0, u+\frac{1}{2} \int_{u-v}^{u+v} \phi(s) d s\right),
$$

which is a line segment.

Note that the plane $y=0$, which is a timelike minimal surface, contains the null line $x= \pm t, y=0$ but they are not a singular curve of the plane.

\section{Reflection principles of generalized timelike surfaces}

Spacelike maximal surfaces have both the geodesic reflection principle and the planar reflection principle [3]. The line must be spacelike, and the plane must be timelike. It turns out that timelike minimal surfaces also possess both of the geodesic and planar reflection principles. But for timelike minimal surfaces, the lines and the planes of reflection could be either spacelike or timelike.

Another big difference between the reflection properties of the spacelike maximal surfaces and the timelike minimal surfaces is that, as we will see in the following lemmas, the reflection properties of the timelike minimal surfaces are true only locally near the line or the plane because of the $C^{2}$ rather than $C^{\omega}$ regularity. That is, a timelike surface may be symmetric near the line or the plane of reflection but it may not be so away from the line or the plane.

\subsection{Regular reflection principles}

Lemma 4.1 (Geodesic reflection principle). Suppose a timelike minimal surface contains (a piece of) a spacelike line or a timelike line. Then the surface is locally symmetric with respect to the line.

Proof. We first consider the case where the line is spacelike. Without loss of generality, we may assume that the line is contained in the $x$-axis. Then, we may reconstruct the surface by the regular Bjölring representation formula ( $c f$. Theorem 3.3) with the following Björling data

$$
\gamma(u)=(u, 0,0), \quad N(u)=(0, \cosh \varphi(u), \sinh \varphi(u)) .
$$

Note that $\mathcal{L}(u)=N(u) \times \dot{\gamma}(u)=(0, \sinh \varphi(u), \cosh \varphi(u))$. If we write the resulting $\mathbf{x}$ as $(x, y, t)$, then we see

$$
x(u, v)=u, \quad y(u, v)=\frac{1}{2} \int_{u-v}^{u+v} \sinh \varphi(s) d s, \quad t(u, v)=\frac{1}{2} \int_{u-v}^{u+v} \cosh \varphi(s) d s .
$$


Hence

$$
x(u,-v)=x(u, v), \quad y(u,-v)=-y(u, v), \quad t(u,-v)=-t(u, v),
$$

and the claim is proved.

The case where the line is timelike is similar to the above.

There exists a timelike minimal surface which contains (a piece of ) a lightlike line, such as the plane $y=0$ for example. If $\mathcal{X}$ in (2.8) is a lightlike line, the resulting surface contains (a piece of) a null line. We do not know what kinds of reflection properties hold true for these kind of surfaces.

Lemma 4.2 (Planar reflection principle). Suppose a timelike minimal surface is perpendicular to a spacelike or a timelike plane. Then the surface is locally symmetric with respect to the plane.

Proof. We first consider the case where the plane is spacelike. Without loss of generality, we may assume that the plane perpendicular to the surface is the $x y$-plane. Then, we may reconstruct the surface by the regular Bjölring representation formula ( $c f$. Theorem 3.3) with the following Björling data

$$
\gamma(u)=(\alpha(u), \beta(u), 0), \quad N(u)=(\cos \varphi(u), \sin \varphi(u), 0)
$$

for some functions $\alpha, \beta$ and $\varphi$. Note that

$$
\left.\mathcal{L}(u)=N(u) \times \gamma^{\prime}(u)=\left(0,0, \alpha^{\prime}(u) \sin \varphi(u)-\beta^{\prime}(u) \cos \varphi(u)\right)\right) .
$$

If we write $\mathbf{x}(u, v)=(x(u, v), y(u, v), t(u, v))$, then we see

$$
\begin{gathered}
x(u, v)=\frac{1}{2}(\alpha(u+v)+\alpha(u-v)), \quad y(u, v)=\frac{1}{2}(\beta(u+v)+\beta(u-v)), \\
t(u, v)=\frac{1}{2} \int_{u-v}^{u+v}\left(\alpha^{\prime}(s) \sin \varphi(s)-\beta^{\prime}(s) \cos \varphi(s)\right) d s .
\end{gathered}
$$

Hence

$$
x(u,-v)=x(u, v), \quad y(u,-v)=y(u, v), \quad t(u,-v)=-t(u, v),
$$

and the claim is proved.

The case where the plane is timelike is similar to the above.

There are timelike minimal surfaces which are perpendicular to lightlike planes. For example the timelike minimal surface $y=0$ is perpendicular to the lightlike plane $x=t$. We do not know what kinds of reflection properties hold true for these kind of surfaces.

Timelike catenoids and helicoids are good examples with the aforementioned reflection properties. 


\subsection{Singular reflection principles}

As for generalized spacelike maximal surfaces, $c f$. [12], generalized timelike minimal surfaces also satisfy reflection principles with respect to a folded singularity or with respect to a collapsed singularity.

Lemma 4.3 (Reflection with respect to a folded singularity). Let $\gamma:(a, b) \rightarrow$ $\mathbb{L}^{3}$ be a $C^{2}$ null curve possibly with isolated vanishing velocities. Then, $\mathbf{x}(u, v):=$ $\frac{1}{2}(\gamma(u+v)+\gamma(u-v))$ is a generalized timelike minimal surface with folding singular points on $I_{a, b} \subset \diamond_{a, b}$, and

$$
\mathbf{x}(u,-v)=\mathbf{x}(u, v) \quad \text { for any }(u, v) \in \diamond_{a, b} .
$$

Proof. The claims follow easily from Theorem 3.5 and the definition of folding singular points.

Lemma 4.4 (Reflection with respect to a shrunken singularity). Let $\mathcal{L}$ : $(a, b) \rightarrow \mathbb{L}^{3}$ be a null vector field with isolated zeros. Then

$$
\mathbf{x}(u, v):=\frac{1}{2} \int_{u-v}^{u+v} \mathcal{L}(s) d s
$$

is a generalized timelike minimal surface with shrinking singular points on $I_{a, b} \subset \diamond_{a, b}$, and

$$
\mathbf{x}(u,-v)=-\mathbf{x}(u, v) \quad \text { for any }(u, v) \in \diamond_{a, b} .
$$

Proof. It follows easily from Theorem 3.5 and the definition of folding singular points.

\subsection{A relation between the reflection principles of conjugate surfaces}

We generalize the definitions in [14] and [15] as follows: Given a generalized timelike minimal surface $\mathcal{X}(x)+\mathcal{Y}(y)$ in terms of T. K. Milnor's representation formula, $c \mathcal{X}(u) \pm \frac{1}{c} \mathcal{Y}(v)$ for $c \in \mathbb{R} \backslash\{0\}$ is an associate family and $\mathcal{X}(x)-\mathcal{Y}(y)$ is the conjugate timelike minimal surface of $\mathcal{X}(x)+\mathcal{Y}(y)$. We remark that this definitions are only local.

At this point, we rewrite the expressions for the timelike catenoids and helicoids in terms of the T. K. Milnor's representation formula and observe that they are conjugate surfaces of each other.

Example 4.5. We substitute $u, v$ by $\frac{x+y}{2}, \frac{x-y}{2}$ for the formula in Example 2.9 of $\mathbf{x}_{\mathrm{T} * \circ}$ where $* \in\{E, P, H\}$ and $\circ \in\{C, H\}$ to see

$\mathbf{x}_{\mathrm{T} * \mathrm{C}}(u, v)=\mathcal{X}_{\mathrm{T} * \mathrm{C}}(u+v)-\mathcal{X}_{\mathrm{T} * \mathrm{C}}(u-v), \quad \mathbf{x}_{\mathrm{T} * \mathrm{H}}(u, v)=\mathcal{X}_{\mathrm{T} * \mathrm{H}}(u+v)+\mathcal{X}_{\text {T*H }}(u-v)$

where the null curves $\mathcal{X}_{\text {T*० }}$ are

$$
\begin{aligned}
\mathcal{X}_{\mathrm{TEC}}(t) & =\frac{1}{2}(\sin t,-\cos t, t), & \mathcal{X}_{\mathrm{TEH}}(t) & =\frac{1}{2}(\cos t, \sin t, t), \\
\mathcal{X}_{\mathrm{TPC}}(t) & =\frac{1}{2}\left(t-\frac{t^{3}}{3}, t^{2}, t+\frac{t^{3}}{3}\right), & \mathcal{X}_{\mathrm{TPH}}(t) & =-\frac{1}{2}\left(t-\frac{t^{3}}{3}, t^{2}, t+\frac{t^{3}}{3}\right),
\end{aligned}
$$




$$
\mathcal{X}_{\mathrm{THC}}(t)=\frac{1}{2}(t, \cosh t, \sinh t), \quad \mathcal{X}_{\mathrm{THн}}(t)=-\frac{1}{2}(t, \cosh t, \sinh t) .
$$

In terms of the Björling representation formulae, we see:

Lemma 4.6. Given two $C^{2}$ null curves $\gamma, L:(a, b) \rightarrow \mathbb{L}^{3}$, the following, when they are generalized timelike minimal surfaces, are the conjugate surfaces of each other.

$$
\begin{aligned}
& \frac{1}{2}(\gamma(u+v)+\gamma(u-v))+\frac{1}{2}(L(u+v)-L(u-v)), \\
& \frac{1}{2}(L(u+v)+L(u-v))+\frac{1}{2}(\gamma(u+v)-\gamma(u-v)) .
\end{aligned}
$$

Proof. Trivial.

We remark that if a generalized timelike surface has shrinking singular points on the $u$-axis, then the conjugate surface has folding singular points on the $u$ axis, and vice versa.

The terminology 'conjugate surface' is to be justified by the following, which is true for minimal surfaces in $\mathbb{E}^{3}$ and for spacelike maximal surfaces in $\mathbb{L}^{3}$.

Lemma 4.7. If a timelike minimal surface contains (a piece of) a line which is spacelike or timelike, then its conjugate surface is perpendicular to a plane normal to the line.

Conversely, if a timelike minimal surface is perpendicular to a spacelike or timelike plane, then its conjugate surface contains (a piece of) a line normal to the plane.

Proof. Consider the first claim when the line is spacelike. Without loss of generality, we may assume that the line is contained in the $x$-axis. Then, we may reconstruct the surface, which we call $\mathbf{x}$, from the regular Björling representation formula in Theorem 3.3 with Björling data

$$
\gamma(u)=(u, 0,0), \quad N(u)=(0, \cosh \varphi(u), \sinh \varphi(u))
$$

for some function $\varphi$. Let $\mathbf{x}_{c}(u, v)=(x(u, v), y(u, v), t(u, v))$ be the conjugate surface of $\mathbf{x}$. Then

$$
\mathbf{x}_{c}(u, v)=\frac{1}{2}(L(u+v)+L(u-v))+\frac{1}{2}(\gamma(u+v)-\gamma(u-v)),
$$

where $L^{\prime}(u)=N(u) \times \dot{\gamma}(u)=(0, \sinh \varphi(u), \cosh \varphi(u))$. We immediately see that $x(u, 0)$ is constant, say $x_{0}$, so the image of the curve $\mathbf{x}_{c}(u, 0)$ lies in the plane $x=x_{0}$. Furthermore, it is easy to see that

$$
\partial_{v} y(u, 0)=0, \quad \partial_{v} t(u, 0)=0,
$$

which finishes the proof of the first claim.

All other cases follow similarly.

The importance of the above lemma is that Karcher's conjugate surface construction seems possible for timelike minimal surfaces. The difficulties for actually carrying out the conjugate construction for spacelike maximal or timelike minimal surfaces may come from the existence of singularities. 


\section{A comparison between spacelike maximal surfaces and timelike minimal surfaces with folded singularities}

In this section, we finally answer the question posed in the introduction through the following theorem.

Theorem 5.1. Suppose that $\gamma:(a, b) \rightarrow \mathbb{L}^{3}$ is analytic and null. Then,

$$
\begin{aligned}
& \mathbf{x}(u, v):=\frac{1}{2}(\gamma(u+v)+\gamma(u-v)), \\
& \mathbf{y}(u, v):=\frac{1}{2}(\gamma(u+i v)+\gamma(u-i v))
\end{aligned}
$$

satisfy the following:

(1) $\mathbf{x}$ is a generalized timelike minimal surface defined on $\nabla_{a, b}$ with folding singular points on $I_{a, b}$,

(2) $\mathbf{y}$ is a generalized spacelike maximal surface defined on $\nabla_{a, b}$ with folding singular points on $I_{a, b}$, and

(3) the images of $\mathbf{x}$ and $\mathbf{y}$ match smoothly along $\gamma$.

Proof. The first two claims follow directly from the results of the previous sections. For the third claim, let's write $\gamma(t)=\sum_{n=0}^{\infty} a_{n} t^{n}$ for some real vectors $a_{n}$ 's of dimension 3 . Then,

$$
\begin{aligned}
& \mathbf{x}(u, v)=\sum_{n=0}^{\infty} \frac{a_{n}}{2}\left((u+v)^{n}+(u-v)^{n}\right), \\
& \mathbf{y}(u, v)=\sum_{n=0}^{\infty} \frac{a_{n}}{2}\left((u+i v)^{n}+(u-i v)^{n}\right) .
\end{aligned}
$$

Now we see that

$$
\begin{aligned}
(u+v)^{n}+(u-v)^{n} & =\sum_{r=0}^{n}\left(\begin{array}{l}
n \\
r
\end{array}\right) u^{r} v^{n-r}\left(1+(-1)^{n-r}\right), \\
(u+i v)^{n}+(u-i v)^{n} & =\sum_{r=0}^{n}\left(\begin{array}{l}
n \\
r
\end{array}\right) u^{r} v^{n-r}\left(1+(-1)^{n-r}\right) i^{n-r} .
\end{aligned}
$$

Since $1+(-1)^{n-r}=\left\{\begin{array}{ll}0 & \text { if } n-r \text { is odd, } \\ 2 & \text { if } n-r \text { is even }\end{array}\right.$ odd powers of $v$ do not appear in the expressions for $\mathbf{x}$ and $\mathbf{y}$, and if $v^{2}$ is replaced by $-v^{2}$ in the expression of $\mathbf{y}$ then the expression for $\mathbf{x}$ is obtained. Furthermore, we see that the graphical function $f$ for $\mathbf{x}$ transits analytically to a graphical function for $\mathbf{y}$.

Replacing $v^{2}$ by $-v^{2}$ is the same as replacing $v$ by $i v$, hence this is another instance of the Wick rotation.

Figure 4 clearly shows that the smooth matching property does not hold true in general for a spacelike maximal surface and a timelike minimal surface given by (2.5) and (3.2) with the same Björling data. 
For generalized timelike minimal and spacelike maximal surfaces with a shrunken singularity which can be written as

$$
\mathbf{x}(u, v)=\frac{1}{2} \int_{u-v}^{u+v} \mathcal{L}(s) d s, \quad \mathbf{y}(u, v)=-\frac{i}{2} \int_{u-i v}^{u+i v} \mathcal{L}(s) d s
$$

for some null vector field $\mathcal{L}$, respectively, we see that

$$
\begin{aligned}
& \partial_{v} \mathbf{x}(u, v)=\frac{1}{2}(\mathcal{L}(u+v)+\mathcal{L}(u-v)), \\
& \partial_{v} \mathbf{y}(u, v)=\frac{1}{2}(\mathcal{L}(u+i v)+\mathcal{L}(u-i v))
\end{aligned}
$$

and that $\partial_{v} \mathbf{x}(u, 0) \equiv \partial_{v} \mathbf{y}(u, 0)$. This means, in terms of the original surfaces $\mathbf{x}$ and $\mathbf{y}$, that the coordinate vector fields $\partial_{v} \mathbf{x}$ and $\partial_{v} \mathbf{y}$ match smoothly. Spacelike and timelike catenoids are good examples with which one can observe the phenomenon we have just discussed.

We would like to remark that, after the completion of this paper, a Björling formula for timelike minimal surfaces was considered independently in [5].

\section{References}

[1] J. A. Aledo and J. A. Gálvez, A Weierstrass representation for linear Weingarten spacelike surfaces of maximal type in the Lorentz-Minkowski space, J. Math. Anal. Appl. 283 (2003), no. 1, 25-45.

[2] J. A. Aledo, J. A. Gálvez, and P. Mira, Björling Representation for spacelike surfaces with $H=c K$ in $\mathbf{L}^{3}$, Proceedings of the II International Meeting on Lorentzian Geometry, Publ. de la RSME 8 (2004), 2-7.

[3] L. J. Alías, R. M. B. Chaves, and P. Mira, Björing problem for maximal surfaces in Lorentz-Minkowski space, Math. Proc. Cambridge Philos. Soc. 134 (2003), no. 2, 289316.

[4] V. I. Arnold, Lectures on Partial Differential Equations, Springer, Berlin, 2004.

[5] R. M. B. Chavez, M. P. Dussan, and M. Magid, Björling problem for timelike surfaces in the Lorentz-Minkowski space, arXiv:0906.2361.

[6] F. Dillen and W. Kühnel, Ruled Weingarten surfaces in Minkowski 3-space, Manuscripta Math. 98 (1999), no. 3, 307-320.

[7] F. J. M. Estudillo and A. Romero, Generalized maximal surfaces in Lorentz-Minkowski space $L^{3}$, Math. Proc. Cambridge Philos. Soc. 111 (1992), no. 3, 515-524.

[8] I. Fernández and F. J. López, Periodic maximal surfaces in the Lorentz-Minkowski space $\mathbb{L}^{3}$, Math. Z. 256 (2007), no. 3, 573-601.

[9] S. Fujimori, W. Rossman, M. Umehara, K. Yamada, and S.-D. Yang, New maximal surfaces in Minkowski 3-space with arbitrary genus and their cousins in de Sitter 3space, Results Math. 56 (2009), no. 1-4, 41-82.

[10] H. Karcher, Construction of Minimal Surfaces, Reports on Global Analysis XIII, Tokyo, 1989.

[11] Y. W. Kim, S.-E. Koh, H. Shin, and S.-D. Yang, Generalized surfaces with constant $H / K$ in Euclidean three-space, Manuscripta Math. 124 (2007), no. 3, 343-361.

[12] Y. W. Kim and S.-D. Yang, Prescribing singularities of maximal surfaces via a singular Bjölring representation formula, J. Geom. Phys. 57 (2007), no. 11, 2167-2177.

[13] O. Kobayashi, Maximal surfaces in the 3-dimensional Minkowski space $\mathbb{L}^{3}$, Tokyo J. Math. 6 (1983), no. 2, 297-309. 
[14] S. Lee, Weierstrass representation for timelike minimal surfaces in Minkowski 3-space, arXiv:math/0608726v2.

[15] T. K. Milnor, Surfaces in Minkowski 3-space on which $H$ and $K$ are linearly related, Michigan Math. J. 30 (1983), no. 3, 309-315.

[16] P. Mira and J. A. Pastor, Helicoidal maximal surfaces in Lorentz-Minkowski space, Monatsh. Math. 140 (2003), no. 4, 315-334.

[17] B. O'Neill, Semi-Riemannian Geometry with Applications to Relativity, Academic Press, New York, 1983.

Young WOOK KIM

Department of Mathematics

Korea UNIVERSiTy

SEOUL 136-701, KoreA

E-mail address: ywkim@korea.ac.kr

SunG-Eun KoH

Department of Mathematics

KONKUK UNIVERSITY

SeOul 143-701, Korea

E-mail address: skoh@konkuk.ac.kr

HEAYONG SHIN

Department of Mathematics

Chung-Ang University

SeOul 156-756, KoreA

E-mail address: hshin@cau.ac.kr

SeOng-Deog Yang

Department of Mathematics

Korea University

Seoul 136-701, Korea

E-mail address: sdyang@korea.ac.kr 